\title{
The Effect of Eccentricity on Searches for Gravitational-Waves from Coalescing Compact Binaries in Ground-based Detectors
}

\author{
Duncan A. Brown ${ }^{1}$, Peter J. Zimmerman ${ }^{1}$ \\ 1 Department of Physics, Syracuse University, Syracuse, NY 13244
}

(Dated: October 27, 2018)

\begin{abstract}
Inspiralling compact binaries are expected to circularize before their gravitational-wave signals reach the sensitive frequency band of ground-based detectors. Current searches for gravitational waves from compact binaries using the LIGO and Virgo detectors therefore use circular templates to construct matched filters. Binary formation models have been proposed which suggest that some systems detectable by the LIGO-Virgo network may have non-negligible eccentricity. We investigate the ability of the restricted 3.5 post-Newtonian order TaylorF2 template bank, used by LIGO and Virgo to search for gravitational waves from compact binaries with masses $M \leq 35 M_{\odot}$, to detect binaries with non-zero eccentricity. We model the gravitational waves from eccentric binaries using the $x$-model post-Newtonian formalism proposed by Hinder et al. [I. Hinder, F. Hermann, P. Laguna, and D. Shoemaker, arXiv:0806.1037 1 1]. We find that small residual eccentricities $\left(e_{0} \lesssim 0.05\right.$ at $40 \mathrm{~Hz}$ ) do not significantly affect the ability of current LIGO searches to detect gravitational waves from coalescing compact binaries with total mass $2 M_{\odot}<M<15 M_{\odot}$. For eccentricities $e_{0} \gtrsim 0.1$, the loss in matched filter signal-to-noise ratio due to eccentricity can be significant and so templates which include eccentric effects will be required to perform optimal searches for such systems.
\end{abstract}

PACS numbers: 04.30.-w, 04.25.Nx, 04.30.Db, 04.80.Nn

\section{INTRODUCTION}

In October 2007 the Laser Interferometer Gravitational-Wave Observatory (LIGO) [1] completed its fifth science run, collecting one year of coincident data at design sensitivity [2]. After a period of detector upgrades [3], LIGO and the French-Italian Virgo detector [4] began a new observing campaign in July 2009 and construction of the Advanced LIGO (AdvLIGO) detectors [5] is underway. The inspiral and coalescence of compact binary systems consisting of neutron stars (NS) and/or black holes (BH) is a promising source of gravitational waves for the LIGO-Virgo network [6, 7]. Optimal searches for inspiral signals use the method of matched-filtering [8, 9], in which the time-series of the detector is cross-correlated against a set of theoretical template waveforms. The utility of this technique rests on how well the template waveforms model the signal being sought. Significant progress has been made in modeling gravitational waves from compact binaries using post-Newtonian (PN) theory [19] and numerical relativity (NR) 11. For binaries that evolved through typical main sequence evolution [12, 13], radiation reaction will cause them to circularize by the time the frequency of their gravitational waves enters the sensitive frequency band of ground-based detectors such as LIGO and Virgo [14, 15]. Alternative formation mechanisms have been proposed that may yield binaries with non-negligible eccentricity in the LIGO band [16, 17]. The scattering of stellar mass BHs in galactic cores containing a super-massive BH may lead to binary formations with high eccentricities; $90 \%$ with $e_{0}>0.9$, where $e_{0}$ denotes the eccentricity of the binary when it enters the sensitive band of the detector [16]. In such an encounter, the two BHs radiate enough energy to form a bound system, but do not fully radiate their eccentricity in GWs until they coalesce. Estimates suggest that the rate of such $\mathrm{BH}$ binary coalescences detectable by AdvLIGO may be as high as $100 \mathrm{yr}^{-1}$ [16]. Binary-binary interactions in the central regions of globular clusters may also be a source of gravitational waves with non-zero eccentricities. Multibody interactions in globular clusters may result in the formation of a stable hierarchical triple. If the orbital planes of the inner and outer binary with respect to the center of mass are highly inclined with respect to one another, Kozai resonance increases the eccentricity of the inner binary [17]. It has been estimated that $\sim 30 \%$ of binaries formed in this way will have eccentricities $e_{0}>0.1$ when they enter the AdvLIGO frequency band $(\sim 10 \mathrm{~Hz})$. However, most template families, including those used by LIGO to search for inspiral signals, make use of the quasi-circular approximation. In this scheme, the binary system evolves secularly through a sequence of circular orbits [18, 19]; eccentricity is neglected.

In recent years there has been significant progress in modeling eccentric binaries using post-Newtonian theory. These calculations use a combination of multi-scale methods and variation of constants [20, 22, 23] to include conservative post-Newtonian effects in the phase evolution [22] in addition to the effect of radiation reaction. The conservative dynamics account for small oscillations in phase evolution which occur on time-scales of the order of an orbital period. At present, the $3 \mathrm{PN}$ conservative contributions to the phase evolution have been derived in the quasi-Keplerian parameterization [24] and the $3 \mathrm{PN}$ reactive contribution is expected shortly now that the calculation of the $3 \mathrm{PN}$ flux [25] is complete.

The first investigation of the effect of eccentricity on searches for gravitational waves was performed by Mar- 
tel and Poisson [26]. In their study, they examined the effect of the leading-order radiation effects [14] and concluded that circular templates were effective at capturing sources with small residual eccentricity. This problem was recently revisited by Cokelaer and Pathak [27] using a discrete template bank in an effort to more accurately model an inspiral search. The qualitative conclusions of Ref. [26] were upheld by Ref. [27], despite small quantitative differences due to a difference in the numerical precision used in the computations [27]. These studies used waveforms computed at leading order; both conservative effects on the waveform evolution and higher order postNewtonian corrections to the phase evolution were omitted. Recent work by Tessmer and Gopakumar, based on templates which include conservative post-Newtonian effects, suggests that circular templates may not be adequate to detect systems with residual eccentricity [28].

In this paper, we investigate whether the waveforms used in current LIGO inspiral searches are adequate to detect gravitational waves from compact binaries with residual eccentricity. To model eccentric signals, we use the post-Newtonian eccentric waveform family proposed by Hinder et al. [29], which has been calibrated against numerical simulations of eccentric BH binaries. In Sec. II we review the waveforms used as eccentric signals and in Sec. III we review the data analysis techniques used. Sec.IV presents our results for the current and AdvLIGO detectors.

\section{ECCENTRIC POST-NEWTONIAN WAVEFORMS}

The effect of gravitational radiation on the orbital motion of eccentric binaries was first calculated by Peters and Mathews [14, 15]. Post-Newtonian corrections to the Peters and Mathews result were first calculated in Ref. 20] and later refined using an improved method of variation of constants in Ref. [22]. The PN equations of motion in Ref. [22] were written in terms of the mean motion $n$ and time eccentricity $e_{t}$ and the $3.5 \mathrm{PN}$ phase evolution has been calculated using these variables [23]. The recent progress in the numerical evolution of $\mathrm{BH}$ binaries now allows post-Newtonian models to be calibrated against the gravitational waves extracted from simulations. Hinder et. al. investigated the agreement of eccentric post-Newtonian waveforms with the gravitational waves extracted from an equal mass BH binary with eccentricity $e_{0}=0.1$ [29]. Based on this study they proposed a modification of the eccentric PN waveforms (called the $x$-model) in which the dynamical quantities are written in terms of a variable related to the orbital frequency $x=(M \omega)^{2 / 3}$ and the time eccentricity $e_{t}$. This waveform gives better agreement to the numerical data than the waveform written in terms of $n$ and $e_{t}$ and so we use it to model gravitational waves from eccentric binaries in our analysis. In the remainder of this section, we review the $x$-model formalism introduced in Ref. [29].

\section{A. Conservative post-Newtonian Dynamics}

The post-Newtonian model of Ref. [29] is more easily understood if we first consider the evolution of two point masses $m_{1}$ and $m_{2}$ in the purely Newtonian case. In the Newtonian case, conservation of energy $E$ and angular momentum $J$ dictate the evolution of the orbital elements. We define the mean motion $n$ in terms of the orbital period $P, n=\frac{2 \pi}{P}$. For Newtonian trajectories on an ellipse $\left(0<e_{0}<1\right), n$ is simply $\sqrt{M / a^{3}}$ where $M=m_{1}+m_{2}$ and $a$ is the semi-major axis ${ }^{1}$. To parameterize the equations we introduce the eccentric anomaly $u$. Written in terms of $u$, the relative orbital separation $r$ and the angular frequency $\dot{\phi}$ take the form

$$
\begin{aligned}
& r=a[1-e \cos u] \\
& \dot{\phi}=\frac{n \sqrt{1-e^{2}}}{[1-e \cos u]^{2}}
\end{aligned}
$$

To complete the dynamical system we relate the eccentric anomaly to the mean motion via the Classical Kepler Equation

$$
l=u-e \sin u
$$

where the mean anomaly $l$ is given by integrating $d l=$ $n d t$. In the absence of radiation reaction, $n$ is timeindependent and the mean anomaly is simply $l=n(t-$ $\left.t_{0}\right)$. These equations form the Keplerian parameterization of a Newtonian orbit. Given initial conditions $\phi_{0} \equiv \phi\left(t_{0}\right)$ and $l_{0} \equiv l\left(t_{0}\right)$ along with numerical values for $e_{0}$ and $u$ we can compute the trajectories of the two particles at any time $t>t_{0}$ by root finding for $u$.

The post-Newtonian dynamics of a system are conservative if they the respect energy and angular momentum conservation ${ }^{2}$. Using the relations given in Ref. 24] for $n$ and $e_{t}$ in terms of $E$ and $J$, one can derive $3 \mathrm{PN}$ quasi-Keplerian equations of motion by introducing three eccentricities, $e_{t}, e_{r}$ and $e_{\phi}$, which account for the variations in the $t, r$, and $\phi$ directions. The addition of higher order conservative PN corrections lead to periastron precession. We let $\Delta \phi$ represent the angle of precession during one period $P$. The angle swept out over consecutive periastron passages is defined

$$
\omega=\frac{2 \pi+\Delta \phi}{P}
$$

[25, 29, 30]. We note that $\omega$ is a constant in the absence of radiation reaction.

The evolution equations in the conservative dynamics

\footnotetext{
${ }^{1}$ We work in units with $G=c=1$.

2 This definition fails at $3.5 \mathrm{PN}$ due to center of mass "recoil". A more rigorous definition is given in Ref. 22].
} 
take the abbreviated form [29]

$$
\begin{aligned}
\frac{r}{M} & =\left(1-e_{t} \cos u\right) x^{-1}+r_{1 \mathrm{PN}}+r_{2 \mathrm{PN}} x \\
& +r_{3 \mathrm{PN}} x^{2}+\mathcal{O}\left(x^{3}\right) \\
M \dot{\phi} & =\frac{\sqrt{1-e_{t}^{2}}}{\left(1-e_{t} \cos u\right)^{2}} x^{3 / 2}+\dot{\phi}_{1 \mathrm{PN}} x^{5 / 2}+\dot{\phi}_{2 \mathrm{PN}} x^{7 / 2} \\
& +\dot{\phi}_{3 \mathrm{PN}} x^{9 / 2}+\mathcal{O}\left(x^{11 / 2}\right) \\
l & =u-e_{t} \sin u+l_{2 \mathrm{PN}} x^{2}+l_{3 \mathrm{PN}} x^{3}+\mathcal{O}\left(x^{4}\right) \\
M i & =M n=x^{3 / 2}+n_{1 \mathrm{PN}} x^{5 / 2}+n_{2 \mathrm{PN}} x^{7 / 2} \\
& +n_{3 \mathrm{PN}} x^{9 / 2}+\mathcal{O}\left(x^{11 / 2}\right)
\end{aligned}
$$

Detailed expressions for the PN coefficients are given in the appendix of Ref. 29]. We note that the PN coefficients $r_{\mathrm{PN}}, \dot{\phi}_{\mathrm{PN}}, \ldots$ are functions of both $e_{t}$ and $u$, whereas $n$ depends on $e_{t}$ alone. Conservative PN trajectories are obtained by first integrating Eq. (8) for $l$, and then numerically root solving Eq. (7) for $u$ at each time step $t$.

\section{B. Radiative post-Newtonian Dynamics}

Radiative post-Newtonian dynamics are needed to describe evolutions over time-scales in which angular momentum and energy are carried away from the system by gravitational radiation. The time dependence of $E$ and $J$ implies that $x$ and $e_{t}$ are no longer integrals of motion. The time variation of $n$ and $e_{t}$ leads to a secular evolution of the orbital parameters. To describe the secular evolution of the orbital elements $\phi, \dot{\phi}, r, \dot{r}$, and $l$, the equations of motion for $x$ and $e_{t}$ must be extended into the non-conservative regime. The $2 \mathrm{PN}$ equations describing the radiative dynamics read ${ }^{3}$

$$
\begin{aligned}
& M \dot{x}=\frac{2 \eta}{15\left(1-e_{t}^{2}\right)^{7 / 2}}\left(96+292 e_{t}^{2}+37 e_{t}^{4}\right) x^{5}+\dot{x}_{1 \mathrm{PN}} x^{6} \\
& +\dot{x}_{1.5 \mathrm{PN}} x^{13 / 2}+\dot{x}_{2 \mathrm{PN}} x^{7}+\mathcal{O}\left(x^{15 / 2}\right), \\
& M \dot{e}_{t}=\frac{-e_{t} \eta}{15\left(1-e_{t}^{2}\right)^{7 / 2}}\left(304+121 e_{t}^{2}\right) x^{4}+\dot{e}_{1 \mathrm{PN}} x^{5} \\
& +\dot{e}_{1.5 \mathrm{PN}} x^{11 / 2}+\dot{e}_{2 \mathrm{PN}} x^{6}+\mathcal{O}\left(x^{13 / 2}\right),
\end{aligned}
$$

where $\eta \equiv \mu / M=m_{1} m_{2} /\left(m_{1}+m_{2}\right)^{2}$ is the symmetric mass ratio. Again, we refer to the appendix of Ref. [29] where the PN coefficients are written out explicitly. In the adiabatic approximation, the PN coefficients are independent of $u$, and are solved independently of the Kepler Equation. We use a fourth order Runge-KuttaFehlberg (RKF-45) with adaptive step-size control 31] to numerically solve for the radiative dynamics at each

\footnotetext{
${ }^{3}$ It is conventional in the gravitational-wave literature to define the radiative $\mathrm{PN}$ order relative the order at which radiation reaction occurs.
}

time $t>t_{0}$. Once $x(t)$ and $e_{t}(t)$ are obtained, we substitute their values into Eq. (8) and solve the ordinary differential equation (ODE) for $l(t)$. The value of $l(t)$ is then equated to the RHS of Eq. (7), giving us our PN Kepler Equation. We then solve the PN Kepler Equation using the Mikkola root finding method [32]. The values of $x, e_{t}$, and $u$ are then substituted into the conservative equations for $r$ and $\dot{\phi}$. The value of $\dot{r}$ is obtained numerically using a five-point stencil method and the phase $\phi$ is numerically integrated using the RKF-45. Repeating this process, we obtain the dynamics $(r, \dot{r}, \phi, \dot{\phi})$ at each time $t>t_{0}$.

\section{Formulation of the Dynamics in The Zero Eccentricity Limit}

In the zero eccentricity limit, the $x$-model phasing formalism reduces to a dynamical system closely resembling those of the Taylor time-domain approximants. The system of ordinary differential equations reduces to

$$
\begin{aligned}
M \dot{x} & =\frac{64 \eta}{5} x^{5}\left\{1-\left(\frac{743 \eta}{336}+\frac{11 \eta^{2}}{4}\right) x+4 \pi x^{3 / 2}\right. \\
& \left.+\left(\frac{34103 \eta}{18144}+\frac{13661 \eta^{2}}{2016}+\frac{59 \eta^{3}}{18}\right) x^{2}\right\} \\
M \dot{e} & =0 \\
M \dot{\phi} & =x^{3 / 2} \\
M \dot{l} & =x^{3 / 2}+3 x^{5 / 2}+\frac{1}{4}(28 \eta-18) x^{7 / 2} \\
& +\left(-7 \eta^{2}-\frac{123 \pi^{2} \eta}{32}+\frac{481 \eta}{4}-\frac{27}{2}\right) x^{9 / 2}
\end{aligned}
$$

The evolution equations of the system in the zero eccentricity limit are very similar to the TaylorT4 approximant. The equations which govern the phase evolution, (Eqs. (11), (13)) are equal to the $2 \mathrm{PN}$ TaylorT4 equations [33]. The eccentric $x$-model formalism differs in that it has an amplitude contribution entering in through Eq. (14). We explore the similarity between the two models in greater depth in Sec. IV], where we discuss overlap calculations.

\section{Eccentric Binary Waveforms}

We now discuss the eccentric waveforms used for signals in the template bank simulations. The leading order time domain gravitational-wave polarizations are given 
by the following expressions:

$$
\begin{aligned}
h_{+}= & \frac{-M \eta}{R}\left\{( 1 + \operatorname { c o s } ^ { 2 } \iota ) \left[\left(\frac{M}{r}+r^{2} \dot{\phi}^{2}-\dot{r}^{2}\right) \cos 2 \phi\right.\right. \\
+ & \left.2 r \dot{r} \dot{\phi} \sin 2 \phi]+\left[\frac{M}{r}-r^{2} \dot{\phi}^{2}-\dot{r}^{2}\right] \sin ^{2} \iota\right\},(15 \mathrm{a}) \\
h_{\times}= & \frac{-2 M \eta}{R} \cos \iota\left\{\left(\frac{M}{r}+r^{2} \dot{\phi}^{2}-\dot{r}^{2}\right) \sin 2 \phi\right. \\
& -2 r \dot{r} \dot{\phi} \cos 2 \phi\},
\end{aligned}
$$

where $R$ is the radial distance of the binary, and $\iota$ is the inclination angle of the orbital plane of the binary measured from the line of sight 21, 34]. When the orbit is circular, final term in Eq. (15a) vanishes. This can be seen from the fact that the radial velocity $\dot{r}$ is zero and the Newtonian equation for the centripetal acceleration is $M / r=r^{2} \dot{\phi}^{2}$. For circular motion the polarizations simplify to

$$
\begin{aligned}
& h_{+}=\frac{-2 M \eta r^{2} \Omega^{2}}{R}\left(1+\cos ^{2} \iota\right) \cos 2 \phi, \\
& h_{\times}=\frac{-4 M \eta r^{2} \Omega^{2}}{R} \cos \iota \sin 2 \phi,
\end{aligned}
$$

where $r$ is the binary separation and $\Omega$ is the orbital frequency. Notice that we are using the dominant harmonic only. Since the post-Newtonian waveforms used here do not capture the merger or ringdown of the signals, we terminate the waveforms at the Schwarzschild innermost stable circular orbit (ISCO) frequency, $f_{\text {isco }}=$ $1 /(6 \sqrt{6} \pi M)$. The waveform $h(t)$ observed at the detector is a linear combination of the + and $\times$ polarizations:

$$
\begin{aligned}
h(t)= & F_{+}(\theta, \varphi, \psi) h_{+}\left(t ; \iota, \phi_{0}\right)+ \\
& F_{\times}(\theta, \varphi, \psi) h_{\times}\left(t ; \iota, \phi_{0}\right),
\end{aligned}
$$

where $F_{+}$and $F_{\times}$are the beam-pattern factors of the detector and $\phi_{0}$ is the azimuthal Euler angle of the source. For ground based interferometers, the beam factors are expressed in terms of the Euler angles of the detector $(\theta, \varphi)$ and the Euler angle of the polarization plane $\psi$. Following the convention of Ref. [35], the expressions for the beam-pattern factors are

$$
\begin{aligned}
F_{+}(\theta, \varphi, \psi) & =-\frac{1}{2}\left(1+\cos ^{2} \theta\right) \cos 2 \varphi \cos 2 \psi \\
& -\cos \theta \sin 2 \varphi \sin 2 \psi, \\
F_{\times}(\theta, \varphi, \psi) & =\frac{1}{2}\left(1+\cos ^{2} \theta\right) \cos 2 \varphi \sin 2 \psi \\
& -\cos \theta \cos 2 \varphi \cos 2 \psi .
\end{aligned}
$$

In computing the waveforms, we set the angles such that the binary is optimally oriented for the + polarization mode; i.e, we set $F_{+}=1$ and $F_{\times}=0$. In Fig. 1 we plot two waveforms of a $(1.4+10) M_{\odot}$ system to illustrate the effect of eccentricity on the waveform. In comparing the two waveforms, we find three major effects induced by eccentricity: (i) amplitude modulation; (ii) decreased duration of the signal; and (iii) increased signal amplitude.


FIG. 1: Gravitational wave signals from a $(1.4+10) M_{\odot}$ binary generated using the $x$-model formalism. The waveforms start at $f_{\mathrm{gw}}=40 \mathrm{~Hz}$ and are terminated at the Schwarzschild ISCO frequency. The top panel shows a non-eccentric waveform and in the bottom panel shows a signal with initial eccentricity $e_{0}=0.4$. The sharp peaks are due to the increase in gravitational radiation that occurs during periastron passage. The three major effects of eccentricity illustrated here are: (i) a decreases the duration of the waveform; (ii) waveform amplitude modulation; and (iii) an overall increase in amplitude.

\section{DATA ANALYSIS}

LIGO's current searches for gravitational waves from neutron star and stellar-mass black hole binaries [7] use the restricted stationary phase approximation to the Fourier transform of 3.5 PN circular waveforms (known as TaylorF2) [36, 37]. These templates are given in the frequency domain by

$$
\tilde{h}(f ; M, \eta)=A(M, \eta) f^{-7 / 6} \Theta\left(f-f_{c}\right) e^{i \Psi(f ; M, \eta)},
$$

where $A(M, \eta)$ is the overall amplitude of the template at a canonical distance (typically $1 \mathrm{Mpc}$ ), $\Theta$ is the Heaviside step function and $f_{c}$ is the upper cut-off frequency, given by the Schwarzschild innermost stable circular orbit (ISCO) frequency, $f_{\text {isco }}=1 /(6 \sqrt{6} \pi M)$. The amplitude is included only at leading order, but the phase $\Psi(f ; M, \eta)$ is computed to $3.5 \mathrm{PN}$ order by 


$$
\begin{aligned}
\Psi(f ; M, \eta) & =2 \pi f t_{C}-2 \phi_{C}-\pi / 4+\frac{3}{128 \eta v^{5}}\left\{1+\left(\frac{3715}{756}+\frac{55}{9} \eta\right) v^{2}-16 \pi v^{3}+\left(\frac{15293365}{508032}+\frac{27145}{504} \eta+\frac{3085}{72} \eta^{2}\right) v^{4}\right. \\
& +\pi\left[\frac{38645}{756}-\frac{65}{9} \eta\right]\left[1+3 \ln \left(\frac{v}{v_{0}}\right)\right]+\left\{\frac{11583231236531}{4694215680}-\frac{640}{3} \pi^{2}-\frac{6848}{21}(\gamma+\ln (4 v))\right. \\
& \left.+\left(-\frac{15335597827}{3048192}+\frac{2255}{12} \pi^{2}\right) \eta+\frac{76055}{1728} \eta^{2}-\frac{127825}{1296} \eta^{3}\right\} v^{6} \\
& \left.+\pi\left[\frac{77096675}{254016}+\frac{378515}{1512} \eta-\frac{74045}{756} \eta^{2}\right] v^{7}\right\}
\end{aligned}
$$

where $v=(\pi M f)^{1 / 3}$ and $\gamma$ is Euler's constant [38, 39]. To search for a signal in detector data, we construct the signal-to-noise ratio $\rho$ from the template $h$ and the calibrated output of the detector $s$ according to

$$
\rho=\frac{1}{\sqrt{\langle h \mid h\rangle}}\langle s \mid h\rangle,
$$

where the inner product is given by

$$
\begin{aligned}
\langle s \mid h\rangle & =2 \int_{0}^{\infty} \mathrm{d} f \frac{\tilde{s}^{*}(f) \tilde{h}(f)+\tilde{s}(f) \tilde{h}^{*}(f)}{S_{n}(f)}, \\
& =4 \operatorname{Re} \int_{0}^{\infty} \mathrm{d} f \frac{\tilde{s}^{*}(f) \tilde{h}(f)}{S_{n}(f)},
\end{aligned}
$$

$\tilde{s}(f)$ denotes the Fourier transform of $s(t)$,

$$
\tilde{s}(f)=\int_{-\infty}^{\infty} \mathrm{d} t e^{-2 \pi i f t} s(t),
$$

and $S_{n}(f)$ is the one-side noise power spectral density (PSD) of the detector. In the presence of Gaussian noise alone, the expectation value of $\rho^{2}$ is unity; large values of $\rho$ indicate that a signal is present in the data. The template parameter $t_{C}$ gives the time of arrival of the signal, and can be searched over using a Fourier transform [40]. Similarly the coalescence phase $\phi_{C}$ can be searched over analytically [41]; these parameters are termed extrinsic parameters. The masses of the signal are not known a priori, however, and so a discrete bank of templates must be constructed [42] and the detector data filtered against each template in the bank. Since the mass parameters $M, \eta$ must be explicitly searched over, they are known as intrinsic parameters. The ambiguity function $\mathcal{A}(\vec{\theta})$ is defined with respect to the template parameters $\vec{\theta}=\left(t_{C}, \phi_{C}, M, \eta\right)$ as

$$
\mathcal{A}(\vec{\theta})=\frac{\langle s \mid h\rangle}{\sqrt{\langle s \mid s\rangle\langle h \mid h\rangle}} .
$$

The maximization of Eq. (25) over the extrinsic template parameters defines the overlap

$$
\begin{aligned}
\mathcal{O}(s, h) & =\max _{t_{C}, \phi_{C}} \frac{\langle s \mid h\rangle}{\sqrt{\langle s \mid s\rangle\langle h \mid h\rangle}}, \\
& =\max _{t_{C}, \phi_{C}}\langle\hat{s} \mid \hat{h}\rangle,
\end{aligned}
$$

[40, 42], where the hat denotes normalization of the template amplitude. The fitting factor $\mathcal{F}$, quantifies loss in signal-to-noise ratio (SNR) due to the filtering of a signal with template that is not an optimal filter [43]. The loss in event rate incurred by non-optimal filtering is proportional to $1-\mathcal{F}^{3}$. For instance, a $3 \%$ decrease in $\mathcal{F}$ gives a loss of approximately $10 \%$ in event rate. The value of $\mathcal{F}$ is obtained by maximizing the overlap function over the intrinsic parameters of the template waveform

$$
\mathcal{F}=\max _{M, \eta t_{t_{C}, \phi_{C}}}\langle\hat{s} \mid \hat{h}\rangle
$$

When constructing a bank, the templates are typically placed such that the loss in signal-to-noise ratio due to mismatch between a template with parameters inside the bank and the nearest bank grid point is no greater than $3 \%$; such a bank has a fitting factor of 0.97 .

In this paper, we use the hexagonal template placement algorithm from the LIGO Algorithm Library (LAL) [44, 45] to place a bank which runs over the mass range $1 \leq m_{1}, m_{2} \leq 34 M_{\odot}$ subject to $M \leq 35 M_{\odot}$ at a fitting factor of 0.97 . Template placement depends on the shape of the noise power spectrum of the detector [42]. We model the current LIGO detectors by an analytic fit to the LIGO noise curve [46], given by

$$
\begin{aligned}
S_{n}(f) & =9.0 \times 10^{-46}\left[(4.49 x)^{-56}\right. \\
& \left.+0.16 x^{-4.52}+0.32 x^{2}+0.52\right] \mathrm{Hz}^{-1},
\end{aligned}
$$

where $x=f / 150 \mathrm{~Hz}$ and $f$ is frequency in Hz. Since the current LIGO detectors (Enhanced LIGO) have the same seismic isolation as the Initial LIGO detectors, they have essentially the same low frequency response. Therefore the Initial LIGO PSD is sufficient to model the Enhanced LIGO detectors for the purpose of this study. The Ad- 
vLIGO PSD [47] is modeled by

$$
\begin{aligned}
S_{n}(f) & =1.60 \times 10^{-49} \\
& {\left[300\left(\frac{f}{15 \mathrm{~Hz}}\right)^{-17}+7\left(\frac{f}{50 \mathrm{~Hz}}\right)^{-6}\right.} \\
& +24\left(\frac{300 x}{90}\right)^{-3.45}-\frac{3.5}{x^{2}} \\
& \left.+110\left(\frac{1.02-1.08 x^{2}+0.54 x^{4}}{1+0.21 x^{2}}\right)\right] \mathrm{Hz}^{-1},
\end{aligned}
$$

where $x=f / 300 \mathrm{~Hz}$. The low-frequency cutoff $f_{0}$ for the templates is $40 \mathrm{~Hz}$ for LIGO and $10 \mathrm{~Hz}$ for AdvLIGO.

To study the effectiveness of the template bank described above to capture signals from eccentric systems, we model eccentric binaries by the waveform given in Eq. (18), which includes $3 \mathrm{PN}$ conservative dynamics and $2 \mathrm{PN}$ radiative dynamics. The binary is chosen to be optimally oriented [34]. We generate an eccentric signal and compute the overlap of the waveform against each template in the bank. The overlap is maximized over the template bank to obtain the best match. Following Ref. [48], we define the effective fitting factor $\overline{\mathcal{F}}$

$$
\overline{\mathcal{F}}=\max _{\hat{h} \in \text { bank }}\langle\hat{s} \mid \hat{h}\rangle \text {. }
$$

The function $\overline{\mathcal{F}}$ provides a measure of how effective the template bank is in capturing a signal $s$ that does not lie exactly in the template bank manifold. The distribution of $\overline{\mathcal{F}}$ allows one to isolate the regions of the physical parameter space where the mismatch is less than the minimal match of the bank.

\section{RESULTS}

\section{A. Initial and Enhanced LIGO}

In this section we present the results of our study for the current LIGO detectors. To understand the degree to which eccentricity affects the the $x$-model waveform, we investigated the overlap as a function of eccentricity using a circular $\left(e_{0}=0\right) x$-model waveform as the template and a $x$-model waveform with non-zero eccentricity as the signal. Fig. 2 shows the LIGO PSD overlap as a function of initial eccentricity for three different mass ratios. The overlaps are more sensitive to eccentricity for smaller mass systems, since the lower mass systems have longer durations which allow for the effects of eccentricity to accumulate. We next investigate overlaps between $x$-model signals and 2 PN TaylorT4 templates. In section IIC, we noted the similarities between the zero eccentricity limit of the $x$-model and the 2 PN TaylorT4 approximation. Comparisons are made with the TaylorT4 at $2 \mathrm{PN}$ since that is the radiative PN order at which the $x$-model has been computed. In Fig. 3 , we plot the LIGO PSD overlap between 2PN TaylorT4

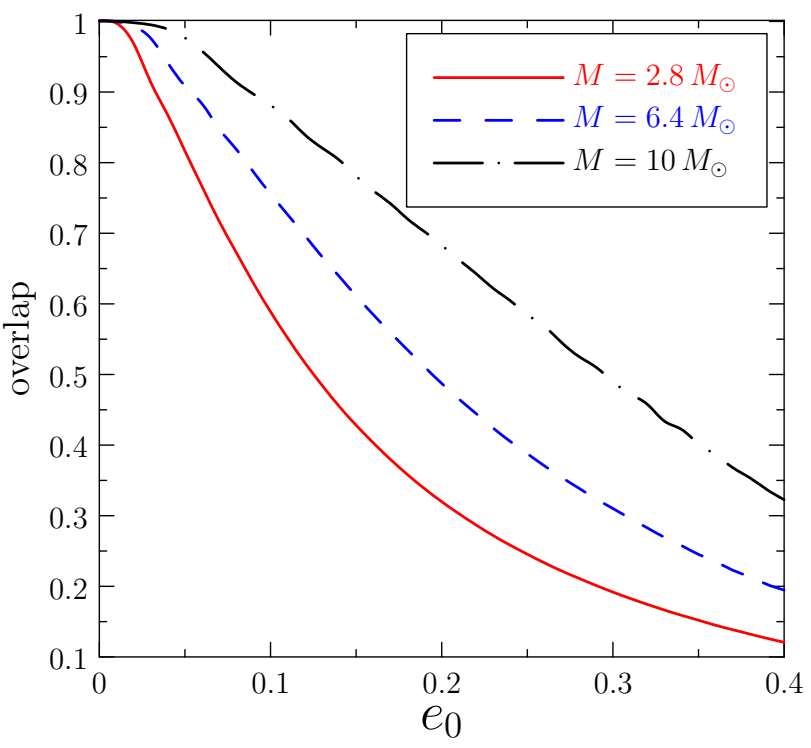

FIG. 2: The overlap $\left\langle\hat{s}_{e=0} \mid \hat{s}\right\rangle$ using the Initial LIGO PSD for three systems having total masses $(1.4+1.4) M_{\odot},(1.4+$ $5.0) M_{\odot}$ and $(5.0+5.0) M_{\odot}$. Here, $s_{e=0}$ denotes the circular $x$-model signal. The overlaps for the higher mass system are less affected by eccentricity because they have fewer cycles in the LIGO frequency band, and can thus be phase-matched against template waveforms via a relative shift in coalescence time without significant phase decoherence.

waveforms and $x$-model waveforms of the same mass as a function of eccentricity. As the eccentricity approaches zero, the overlaps between the T4 and the $x$-model approach unity for all three signal masses, consistent with the zero eccentricity limit of the $x$-model in Sec. IIC.

Fig. 4) shows overlaps for Initial LIGO between 2 PN $x$ model waveforms and 3.5 PN TaylorF2 templates. The overlap is worse for these waveforms, as would be expected. At $e_{0}=0$ the reduction in overlap is due to the difference between the PN models used to generate waveforms used as signals and templates. As $e_{0}$ increases, the figure shows the additional loss in overlap due to the effect of eccentricity. The fact that the overlaps are not unity at $e_{0}=0$ is due to the differences between the PN formulation of the time-domain $x$-model signal, which contains radiative effects to $2 \mathrm{PN}$ order, and the frequency-domain TaylorF2 template, which contains radiative effects to $3.5 \mathrm{PN}$ order. Although higher-order post-Newtonian effects are more significant (at a given frequency) for higher-mass binaries, more massive binaries coalesce at lower frequencies. At $e_{0}=0$, the lowest overlap is observed in $M=2.8 M_{\odot}$ signal. Although higher order post-Newtonian corrections are small for this system, they have many waveform cycles over which to accumulate, resulting in a reduction of overlap. Note, however, that in calculating the overlaps shown in Figs. 4 and 5 the intrinsic parameters are kept fixed. Below we show the effect of maximizing the overlap over a bank, as is the case in a real search. As $e_{0}$ increases, eccentric- 


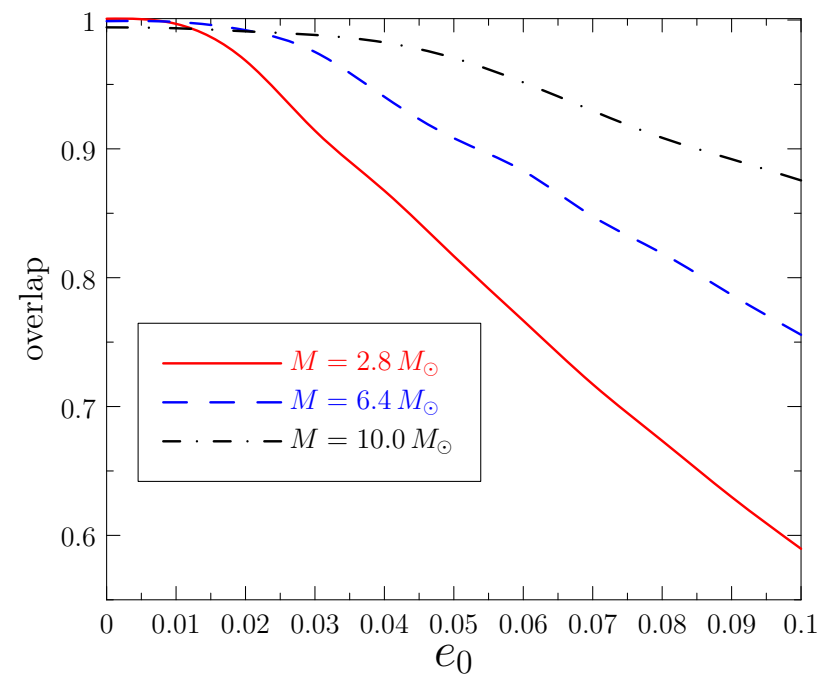

FIG. 3: Overlaps using the LIGO PSD between the $2 \mathrm{PN}$ $x$-model waveform and the $2 \mathrm{PN}$ TaylorT4 waveform of the same total mass for masses $(1.4+1.4) M_{\odot},(1.4+5.0) M_{\odot}$ and $(5.0+5.0) M_{\odot}$. The overlaps approach one as the eccentricity goes to zero, which indicates that the two models are in good agree in the limit $e_{0} \rightarrow 0$.

ity causes additional higher-order post-Newtonian corrections to the signal waveform, but also causes the duration of the waveform to decrease. In the $M=6.8 M_{\odot}$ and $M=10 M_{\odot}$ waveforms, competition between these effects causes the overlap curves to cross at $e_{0}=0.05$, with slightly larger overlaps in the higher-mass signal at larger eccentricity.

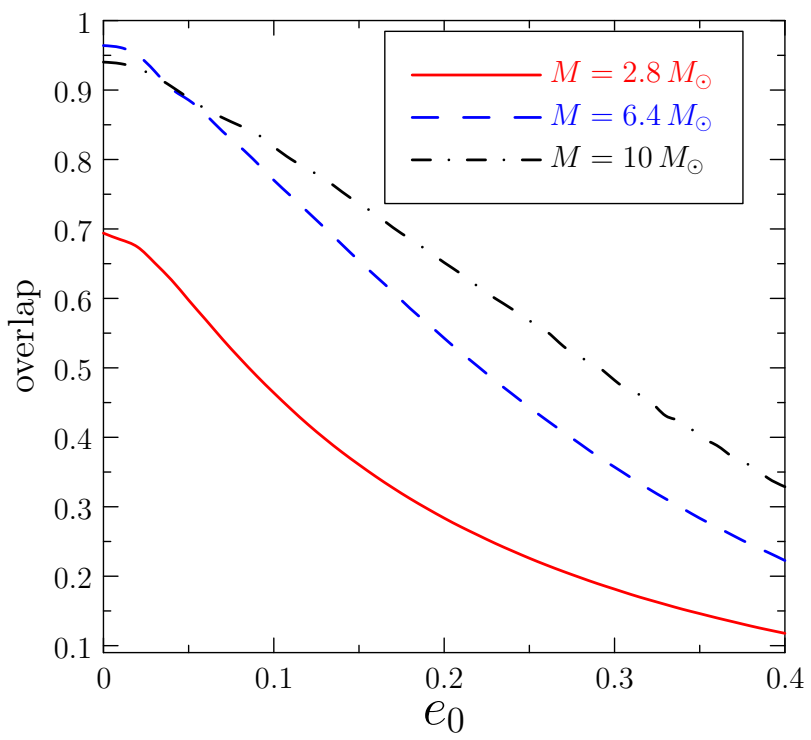

FIG. 4: Initial LIGO overlaps between the $2 \mathrm{PN} x$-model and the 3.5 PN TaylorF2 waveforms as a function of initial eccentricity for $(1.4+1.4) M_{\odot},(1.4+5.0) M_{\odot}$ and $(5.0+5.0) M_{\odot}$ systems. Notice that the $e_{0}=0$ overlaps are not unity. This is due to differences in the $\mathrm{PN}$ order of the waveforms
We now explore the effectiveness of the template bank described in Sec. III at detecting eccentric signals. To explore this for a range of signal masses and eccentricities, we generate a rectangular grid of $10026 x$-model signals with masses in the range $1 M_{\odot} \leq m_{1}, m_{2} \leq 14 M_{\odot}$ subject to $2 \leq M / M_{\odot} \leq 15$ and initial eccentricities in the range $0 \leq e_{0} \leq 0.4$. Each signal in this grid is filtered through the 14863 templates in the 3.5 PN TaylorF2 Initial LIGO template bank. The effective fitting factor $\overline{\mathcal{F}}$ of the bank is computed for each signal. The signal space is three dimensional as it depends on $m_{1}, m_{2}$, and $e_{0}$, and hence $\overline{\mathcal{F}}$ is a function of three variables. To visualize the results of the bank simulation, we plot $\overline{\mathcal{F}}$ as a function of the total mass $M$ and eccentricity $e_{0}$ of the injected $x$-model signal. In our signal grid, several component masses have the same total mass and so in Fig. 6, we plot the highest, lowest, and mean $\overline{\mathcal{F}}$ for each value of $M$. Notice that by maximizing over the template banks, we have gained significantly in overlap for the lower mass systems (cf. Fig. (4). In the worst case, the 3.5 PN TaylorF2 bank can achieve effective fitting factors $\geq 0.96$ for all $x$-model signals which have a total mass $M \geq 3 M_{\odot}$ and an eccentricity $e_{0} \leq 0.05$ at $40 \mathrm{~Hz}$. The signals with the worst values of $\overline{\mathcal{F}}$ are those which are matched against templates which lie near the edges of the template bank. To separate the effect of eccentricity from effects related to the difference in PN order between the signal and template we calculate the relative difference between the effective fitting factor for signals with zero eccentricity and those with non-zero eccentricity, given by

$$
\Delta \overline{\mathcal{F}}\left(e_{0}\right)=\frac{\overline{\mathcal{F}}\left(e_{0}=0\right)-\overline{\mathcal{F}}\left(e_{0}\right)}{\overline{\mathcal{F}}\left(e_{0}=0\right)} .
$$

Fig. 7 shows $\Delta \overline{\mathcal{F}}$ for the highest, lowest, and average values of $\overline{\mathcal{F}}$. These distributions illustrate that the loss in overlap results predominantly from eccentricity at higher masses, but at lower masses is the accumulation of higher-order PN effects (included in the templates, but not in the signals) over the longer waveforms. We therefore conclude that the current LIGO template bank is effective at capturing signals with small residual eccentricities $e_{0} \lesssim 0.05$ at $40 \mathrm{~Hz}$ for all masses $2 \leq M \leq 15 M_{\odot}$. For eccentricities higher than $e_{0} \sim 0.05$, the loss in $\overline{\mathcal{F}}$ becomes more significant, although for the higher mass systems this loss does not occur until $e_{0} \gtrsim 0.1$.

\section{B. Results for Advanced LIGO}

Finally, we investigate how eccentricity effects the detection efficiency of TaylorF2 templates for AdvLIGO. Although it is unlikely that TaylorF2 templates will be used for AdvLIGO searches, this study is illustrative of the effect of eccentricity on circular templates with the AdvLIGO noise curve. The lower frequency cut-off for the AdvLIGO PSD requires that we start our waveforms at $10 \mathrm{~Hz}$ instead of $40 \mathrm{~Hz}$. Fig. 5 shows the overlap 




FIG. 5: Overlaps between the $2 \mathrm{PN} x$-model eccentric waveforms and the $3.5 \mathrm{PN}$ TaylorF2 waveforms using the AdvLIGO PSD. Reduced overlaps result from the increase in the amount of time spent in the detector's band. The increase in the duration leads to larger phase de-coherence and intensifies the effects due to differences in PN order. Additionally, the difference in the PN order of the signals and the templates reduces the overlap even at $e_{0}=0$.

between the 3.5 PN TaylorF2 waveforms and the eccentric $x$-model waveforms for the AdvLIGO noise curve. Since the waveforms have more detectable cycles in AdvLIGO, a larger phase difference accumulates leading to lower overlaps than for Initial LIGO. The effect of the post-Newtonian differences between the waveform and templates is also apparent in Fig. [5, as in Fig. 4,

Our analysis of the AdvLIGO template bank results proceeds in a similar way to that for the current detectors. Due to the improved low-frequency sensitivity of AdvLIGO, the size of the template bank increases to 136000 , greatly increasing the computational cost of the template bank simulation. As a result, we decrease the resolution of the signal grid to inject only 280 signals. This is sufficient to probe the effect of eccentricity on AdvLIGO searches, however. Fig. 8 shows the best, worst and mean values of $\overline{\mathcal{F}}$. Notice that $\overline{\mathcal{F}}$ is very poor for the long, low-mass signals, and the high-mass signals (where higher order PN effects dominate) even at zero eccentricity. This is due to the difference in PN order between the signals and the templates. In Fig. 9 we plot $\Delta \overline{\mathcal{F}}$ for the highest, lowest, and average values of $\overline{\mathcal{F}}$ for AdvLIGO. In the AdvLIGO case, the effect of small residual eccentricity can be significant for low mass systems $\left(M \lesssim 4 M_{\odot}\right)$. Further studies using both higher
PN-order eccentric waveforms and the actual AdvLIGO search templates are needed to confirm these findings.

\section{CONCLUSION}

In this paper we studied the effect of eccentricity on detection searches for gravitational waves from compact binary coalescence using the current and Advanced LIGO detectors. To construct eccentric binary waveforms, we used the NR-calibrated 2 PN $x$-model formalism proposed by Hinder et al. [29]. We considered the zero eccentricity limit of the $x$-model and compared it with the TaylorT4 approximant; analytic and overlap calculations confirm that the phase evolutions of these two models agree in the $e_{0}=0$ limit. Using $x$-model waveforms as our signal family, we performed template bank simulations using the 3.5 PN TaylorF2 waveforms and the hexagonal placement algorithm implemented in LAL [45]. The 3.5 PN TaylorF2 template bank was found to give fitting factors of 0.96 or better for systems with total mass $M \gtrsim 3 M_{\odot}$ and eccentricity at $40 \mathrm{~Hz}$ of $e_{0} \lesssim 0.05$. By separating the loss in fitting factor due to differences in higher-order circular PN corrections and the effect of eccentricity, we conclude that current LIGO searches are sensitive to binaries with small residual eccentricities when the waves enter the sensitive band of the detector. However for eccentricities $e_{0} \gtrsim 0.1$, significant losses in sensitivity will be observed. For AdvLIGO, our results were dominated by differences in the PN order of the signals and templates and so more investigation with higher-order PN eccentric signals is needed. However, our study suggests that AdvLIGO is even more sensitive to the effect of residual eccentricity in compact binary inspiral, as would be expected.

In this study, we did not consider the ability of the LIGO detectors to measure eccentricity, however our results suggest that for $e_{0} \gtrsim 0.1$, LIGO is sensitive to eccentricity, even in the current detectors. Careful study of parameter estimation and implementation of a search for eccentric systems with Enhanced LIGO or AdvLIGO would allow the formation mechanisms proposed in Refs. [16, 17] to be explored.

\section{Acknowledgments}

We thank Ian Hinder, Eric Poisson, and Deirdre Shoemaker for helpful discussions on the subject of postNewtonian modeling of eccentric binaries, Andrew Lundgren and Larne Pekowsky for their contributions to the template bank simulation code and Sukanta Bose for comments on this manuscript. This work was supported by NSF grant PHY-0847611.
[1] B. C. Barish and R. Weiss, Phys. Today 52N10, 44 (1999).
[2] B. P. Abbott et al., Reports on Progress in Physics 72, 

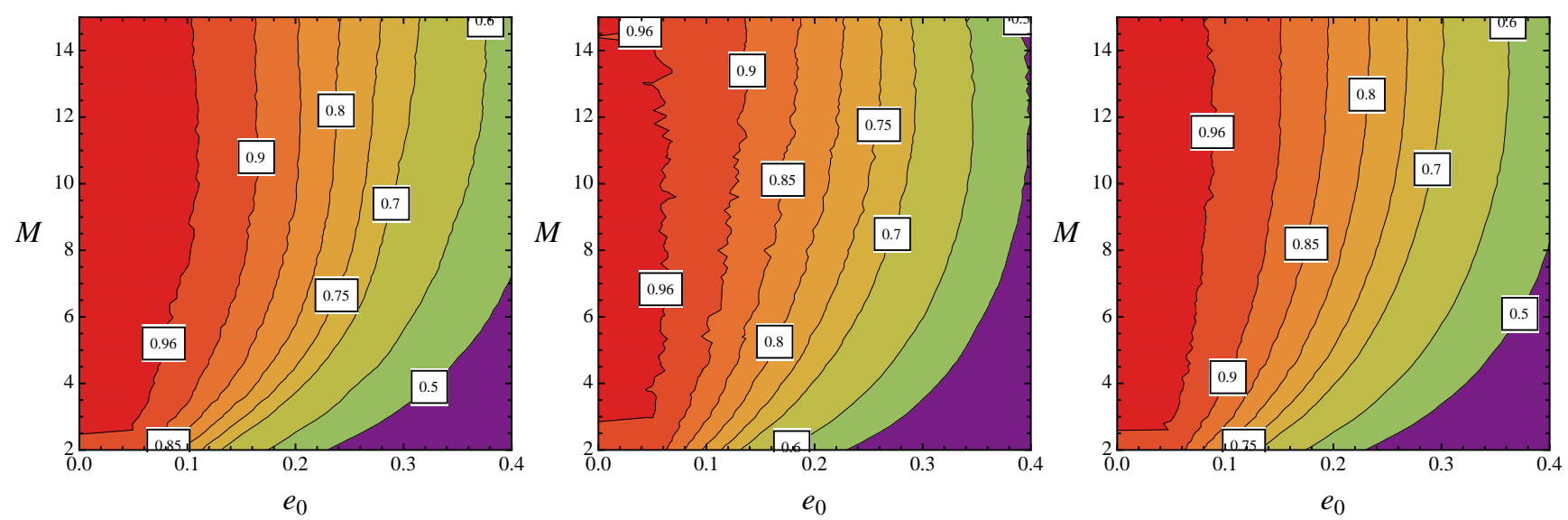

FIG. 6: From left to right the figures show the best, worst and mean value of the effective fitting factor $\overline{\mathcal{F}}$ for the initial LIGO 3.5 PN TaylorF2 bank as a function of the total mass $M$ in solar masses and the eccentricity $e_{0}$ at $40 \mathrm{~Hz}$ of the injected signal. The contours are labeled by the values $\overline{\mathcal{F}}$ enclosed.
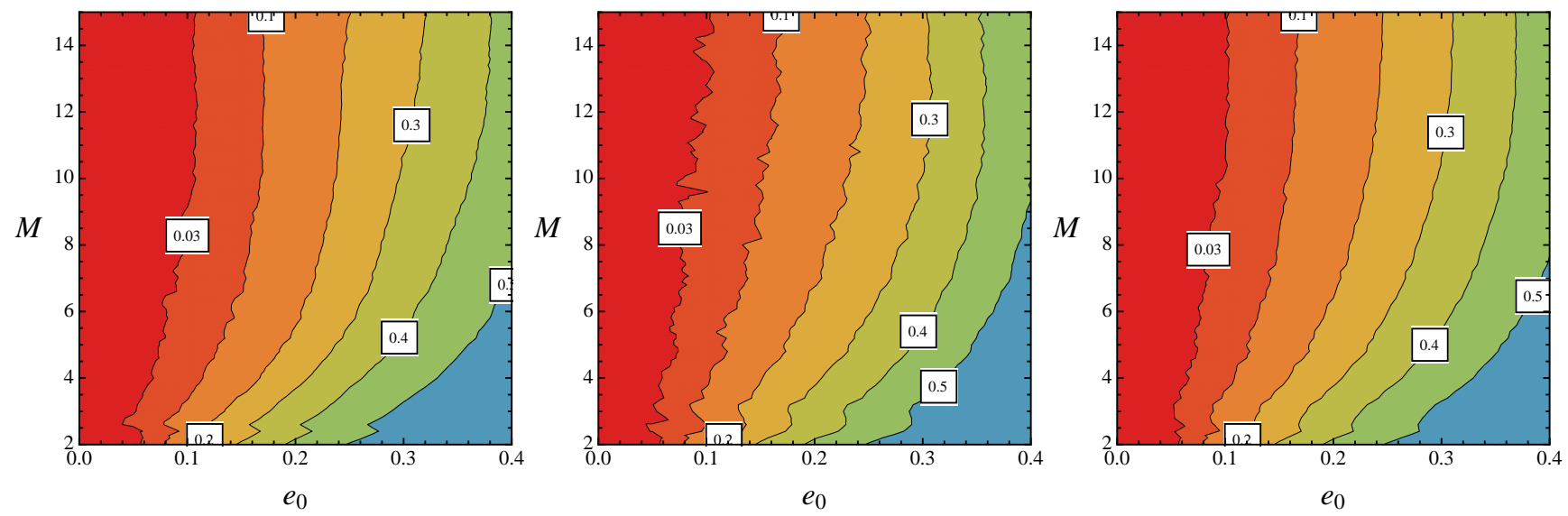

FIG. 7: From left to right the figures show the best, worst and mean value of $\Delta \overline{\mathcal{F}}$ for the Initial LIGO 3.5 PN TaylorF2 bank as a function of the total mass $M$ in solar masses and the eccentricity $e_{0}$ at $40 \mathrm{~Hz}$ of the injected. The contours are labeled by the values $\Delta \overline{\mathcal{F}}$ enclosed.

$076901(2009)$.

[3] J. R. Smith (LIGO Scientific), Class. Quant. Grav. 26, 114013 (2009).

[4] F. Acernese et al. (VIRGO), AIP Conf. Proc. 794, 307 (2005).

[5] P. Fritschel (2003), gr-qc/0308090.

[6] B. P. Abbott et al. (LIGO Scientific) (2009), grqc/0901.0302.

[7] B. P. Abbott et al. (LIGO Scientific) (2009), grqc/0905.3710.

[8] L. Wainstein and V. Zubakov, Extraction of Signals From Noise (Prentice-Hall, 1962).

[9] C. Helstrom, Statistical Theory of Signal Detection (Pergamon-Press, 1968), 2nd ed.

[10] L. Blanchet, Living Rev. Rel. 5, 3 (2002), gr-qc/0202016.

[11] M. Hannam, Class. Quant. Grav. 26, 114001 (2009).

[12] V. Kalogera, C. Kim, D. R. Lorimer, M. Burgay, N. D'Amico, A. Possenti, R. N. Manchester, A. G. Lyne, B. C. Joshi, M. A. McLaughlin, et al., Astrophys. J. 601, L179 (2004), erratum-ibid. 614 (2004) L137.

[13] V. Kalogera et al., Astrophys. J. Lett. 614, L137 (2004).
[14] P. C. Peters, Phys. Rev. 136, B1224 (1964).

[15] P. C. Peters and J. Mathews, Phys. Rev. 131, 435 (1963).

[16] R. M. O'Leary, B. Kocsis, and A. Loeb (2008), astro$\mathrm{ph} / 0807.2638$.

[17] L. Wen, Astrophys. J. 598, 419 (2003).

[18] T. Damour, B. R. Iyer, and B. S. Sathyaprakash, Phys. Rev. D63, 044023 (2001).

[19] L. Blanchet, Living Rev. Rel. 9, 4 (2006).

[20] A. Gopakumar and B. R. Iyer, Phys. Rev. D56, 7708 (1997).

[21] A. Gopakumar and B. R. Iyer, Phys. Rev. D65, 084011 (2002), gr-qc/0110100.

[22] T. Damour, A. Gopakumar, and B. R. Iyer, Phys. Rev. D70, 064028 (2004).

[23] C. Konigsdorffer and A. Gopakumar, Phys. Rev. D73, 124012 (2006).

[24] R.-M. Memmesheimer, A. Gopakumar, and G. Schaefer, Phys. Rev. D70, 104011 (2004).

[25] K. G. Arun, L. Blanchet, B. R. Iyer, and M. S. S. Qusailah (2007), gr-qc/0711.0302.

[26] K. Martel and E. Poisson, Phys. Rev. D60, 124008 

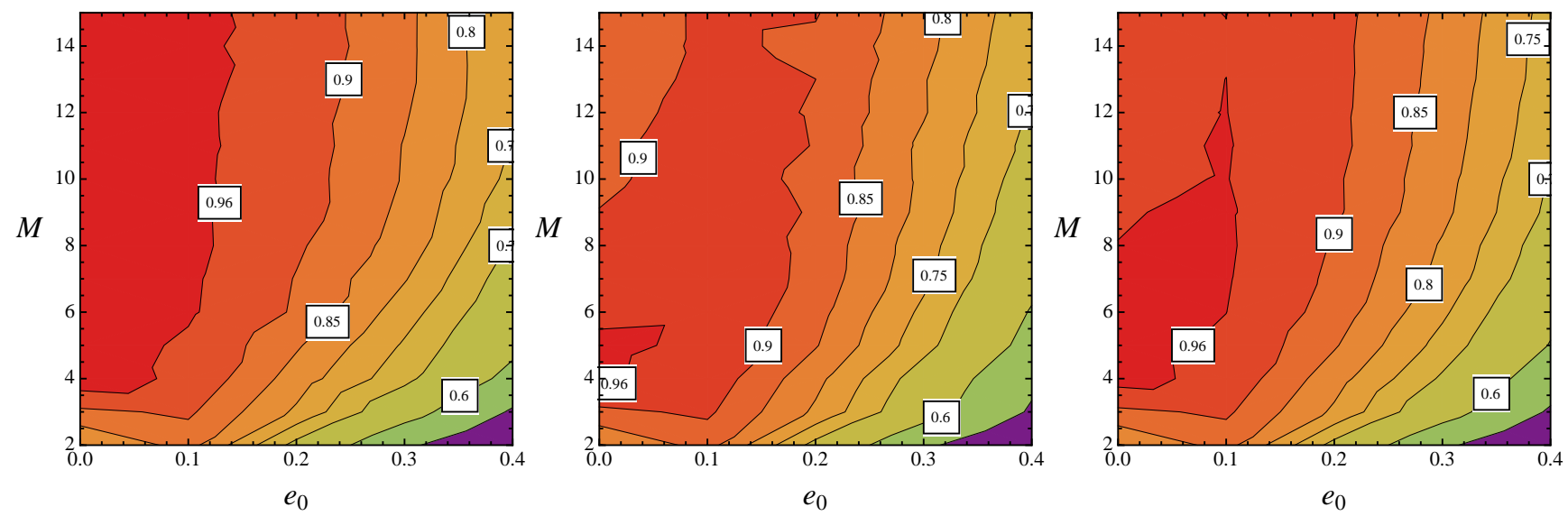

FIG. 8: From left to right the figures show the best, worst and mean value of the effective fitting factor $\overline{\mathcal{F}}$ for the AdvLIGO 3.5 PN TaylorF2 bank as a function of the total mass $M$ in solar masses and the eccentricity $e_{0}$ at $40 \mathrm{~Hz}$ of the injected signal. The contours are labeled by the values $\overline{\mathcal{F}}$ enclosed.
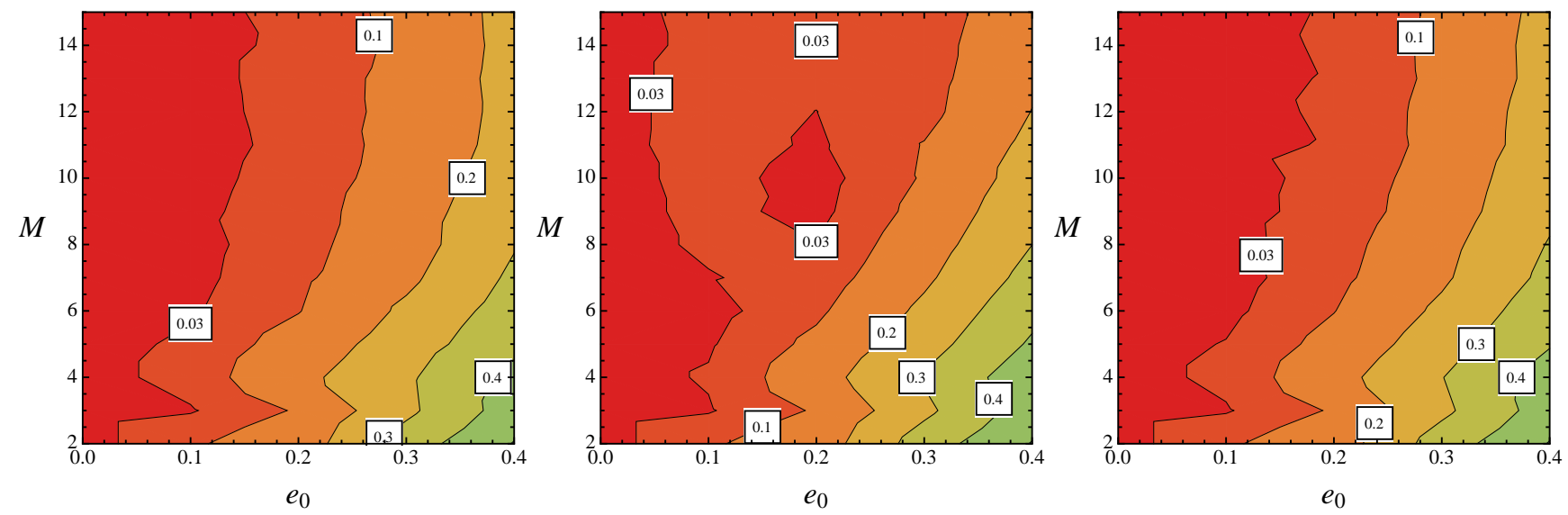

FIG. 9: From left to right the figures show the best, worst and mean value of $\Delta \overline{\mathcal{F}}$ for the AdvLIGO 3.5 PN TaylorF2 bank as a function of the total mass $M$ in solar masses and the eccentricity $e_{0}$ at $40 \mathrm{~Hz}$ of the injected signal. The contours are labeled by the values $\Delta \overline{\mathcal{F}}$ enclosed.

(1999).

[27] T. Cokelaer and D. Pathak, Class. Quant. Grav. 26, 045013 (2009).

[28] M. Tessmer and A. Gopakumar, Phys. Rev. D78, 084029 (2008).

[29] I. Hinder, F. Herrmann, P. Laguna, and D. Shoemaker (2008), gr-qc/0806.1037.

[30] K. G. Arun, L. Blanchet, B. R. Iyer, and M. S. S. Qusailah (2007), gr-qc/0711.0250.

[31] GNU Scientific Library,
http://www.gnu.org/software/gsl/.

[32] S. Mikkola, Celestial Mechanics 40, 329 (1987).

[33] A. Buonanno, B. Iyer, E. Ochsner, Y. Pan, and B. S. Sathyaprakash (2009), gr-qc/0907.0700.

[34] K. S. Thorne (1987), in Hawking, S.W. (ed.), Israel, W. (ed.): Three hundred years of gravitation, 330-458.

[35] W. G. Anderson, J. T. Whelan, P. R. Brady, J. D. E. Creighton, D. Chin, and K. Riles, Tech. Rep. LIGO-T010110-00-Z, LIGO Project (2001), URL http://www.ligo.caltech.edu/docs/T/T010110-00.pdf

[36] C. C and F. E, Phys. Rev. D 49, 2658 (1994).
[37] S. Droz, D. J. Knapp, E. Poisson, and B. J. Owen, Phys. Rev. D 59, 124016 (1999).

[38] L. Blanchet, G. Faye, B. R. Iyer, and B. Joguet, Phys. Rev. D65, 061501 (2002).

[39] L. Blanchet, T. Damour, G. Esposito-Farese, and B. R. Iyer, Phys. Rev. Lett. 93, 091101 (2004).

[40] B. J. Owen, Phys. Rev. D53, 6749 (1996).

[41] B. Allen, W. G. Anderson, P. R. Brady, D. A. Brown, and J. D. E. Creighton (2005), gr-qc/0509116.

[42] B. J. Owen and B. S. Sathyaprakash, Phys. Rev. D60, 022002 (1999).

[43] T. A. Apostolatos, C. Cutler, G. J. Sussman, and K. S. Thorne, Phys. Rev. D49, 6274 (1994).

[44] S. Babak, R. Balasubramanian, D. Churches, T. Cokelaer, and B. S. Sathyaprakash, Class. Quant. Grav. 23, 5477 (2006).

[45] LSC Algorithm Library, URL https://www.lsc-group.phys.uwm.edu/daswg/projects/lal.html.

[46] A. Lazzarini and R. Weiss, LIGO science requirements document (1995), LIGO-E950018-02-E; See also, URL http://www.ligo.caltech.edu/ jzweizig/distribution/LSC_Data 
[47] GWINC: Gravitational Wave Interferometer Noise Calculator, v1 default parameters, URL http://lhocds.ligo-wa.caltech.edu:8000/advligo/GWINC
[48] L. Lindblom, B. J. Owen, and D. A. Brown, Phys. Rev. D78, 124020 (2008). 